\title{
Modified Tangential Frequency Filtering Decomposition and its Fourier Analysis
}

\author{
Qiang Niu — Laura Grigori — Pawan Kumar — Frédéric Nataf
}

\section{$\mathrm{N}^{\circ} 6662$}

September 2008

Thème NUM

\section{apport}

de recherche 



\title{
Modified Tangential Frequency Filtering Decomposition and its Fourier Analysis
}

\author{
Qiang Niu团, Laura Grigor讨, Pawan Kumar团, Frédéric Nataf $\$$ \\ Thème NUM - Systèmes numériques \\ Équipes-Projets Grand-Large \\ Rapport de recherche $n^{\circ} 6662$ - September 2008 - 24 pages
}

\begin{abstract}
In this paper, a modified tangential frequency filtering decomposition (MTFFD) preconditioner is proposed. The optimal order of the modification and the optimal relaxation parameter are determined by Fourier analysis. With this choice of the optimal order of modification, the Fourier results show that the condition number of the preconditioned matrix is $\mathcal{O}\left(h^{-\frac{2}{3}}\right)$, and the spectrum distribution of the preconditioned matrix can be predicted by the Fourier results. The performance of MTFFD is compared with tangential frequency filtering (TFFD) preconditioner on a variety of large sparse matrices arising from the discretization of PDEs with discontinuous coefficients. The numerical results show that the MTFFD preconditioner is much more efficient than the TFFD preconditioner.
\end{abstract}

Key-words: preconditioner; linear system; tangential frequency filtering decomposition; GMRES

* School of Mathematical Sciences, Xiamen University, Xiamen, 361005, P.R. China; The work of this author was performed during his visit to INRIA, funded by China Scholarship Council; (Email:kangniu@gmail.com)

$\dagger$ INRIA Saclay - Ile de France, Laboratoire de Recherche en Informatique Universite Paris-Sud 11, France (Email:laura.grigori@inria.fr).

¥ INRIA Saclay - Ile de France, Laboratoire de Recherche en Informatique Universite Paris-Sud 11, France (Email:kumar@lri.fr).

$\S$ Laboratoire J. L. Lions, CNRS UMR7598, Universite Paris 6, France; (Email: nataf@ann.jussieu.fr)

Centre de recherche INRIA Saclay - Île-de-France

Parc Orsay Université

4, rue Jacques Monod, 91893 ORSAY Cedex

Téléphone : +33172925900 


\section{Préconditionnement à base de filtrage tangentiel modifié et son analyse de Fourier}

Résumé : Dans ce papier nous proposons une modification du préconditionnement à base de filtrage tangentiel (MTFFD). Les valeurs optimales des paramètres de la modification sont déterminées par une analyse de Fourier. Avec ce choix des paramètres, l'analyse de Fourier montre que le conditionnement de la matrice préconditionnée est de l'ordre de $\mathcal{O}\left(h^{-\frac{2}{3}}\right)$. Les résultats numériques présentés montrent que MTFFD est plus efficace que le préconditionnement à base de filtrage tangentiel TFFD.

Mots-clés : préconditionnement, systèmes linéaires, GMRES 


\section{Introduction}

In this paper, we investigate preconditioning techniques for solving the linear system

$$
\mathbf{A x}=\mathbf{b}
$$

with

$$
\mathbf{A}=\left[\begin{array}{cccc}
D_{1} & U_{1} & & \\
L_{1} & D_{2} & \ddots & \\
& \ddots & \ddots & U_{n_{x}-1} \\
& & L_{n_{x}-1} & D_{n_{x}}
\end{array}\right] \in \mathcal{R}^{N \times N}, \quad \mathbf{b} \in \mathcal{R}^{N}
$$

which often arises from the discretization of many PDEs by finite difference or finite volume schemes. When preconditioned iterative methods are used for solving (1), the convergence rate of an iterative method heavily depends on the property of the preconditioner 36. Therefore, developing efficient preconditioners has been one of the major research interests in many applications. Algebraic multigrid (AMG) methods work well for many problems in practice 21. 35. However, conventional AMG methods may suffer from relatively expensive setup time and large memory requirements, particularly for three dimensional problems [18. Another more general preconditioner is the multilevel incomplete block factorization [9] 37. A theoretical comparison of algebraic multigrid methods and algebraic multilevel methods are carried out by Y. Notay 33 for symmetric positive definite (SPD) matrices, and generalized by $\mathrm{C}$. Mense and R. Nabben 29, 30 for nonsymmetric matrices.

Tangential Frequency Filtering Decomposition (TFFD) proposed in [2] is a special kind of incomplete block factorization preconditioner. Similar to some popular preconditioning techniques discussed in [1, 3, 8, 12, 13, 38, 39, 40, 42, the TFFD preconditioner can be used as a preconditioner or a smoother for multigrid methods. The preconditioner has the feature of filtering property, i.e. $(\mathbf{M}-\mathbf{A}) \mathbf{f}=0$ for a vector $\mathbf{f}$, where $\mathbf{M}$ is the TFFD preconditioner. For $\mathbf{A} \succeq 0$ (symmetric positive semidefinite), the preconditioner satisfies $\mathbf{M}-\mathbf{A} \succeq 0$, i.e. $\mathbf{M}$ is a compensative matrix of $\mathbf{A}$ [4, 5, 24. This is an important property that preconditioners of SPD coefficient matrix should possess. By combining TFFD and ILU(0) in a multiplicative way [2, the combinative preconditioner is shown to be very efficient on several challenging problems. Therefore, it is important to give a deep understanding of the TFFD preconditioner. In [7, 8], the authors have presented some nice ways to analyze the properties of general block factorization preconditioners. For frequency filtering decomposition type preconditioners, some analysis have been done in [1, 13, 14, 38, 39. The bounds on the spectral radii or condition numbers are derived by a couple of complicated inequalities. These results are useful in understanding the behavior of the preconditioners. However, the results have difficulty in outlining the range and clustering of the spectrum of the preconditioned matrix, especially when the matrix dimension is large. Fourier analysis is a powerful tool for analyzing the properties of a preconditioner [15]. It has been applied successfully to local model analysis in multigrid methods 10, 11, 41, and popularized by T. F. Chan and H. C. Elman [15] for analyzing algebraic preconditioners and classical iterative methods. Fourier analysis has been recognized as a standard tool for estimating the convergence rate of preconditioned iterative methods, see e.g. T. F. Chan et.al 16, 17, R J. Le Veque and L. N. Trefethen 23, K. Otto 34]. For 
point-wise incomplete factorization type preconditioners like ILU(0), MILU(0) and RILU preconditioners, Fourier analysis has been done in [15, 16, 17. The Fourier analysis of block ILU and MILU factorization preconditioners is considered in 34 for a time-dependent hyperbolic PDE problem.

The original aim of the present work is to analyze the TFFD preconditioner by means of Fourier analysis. Whereas, later we find that Fourier analysis for TFFD preconditioner is not feasible for our model problem. This is because of an exact cancelation in the denominator of a parameter, which is determined by symbolic computation (this will be shown in Section 3). But this does not mean that in practice TFFD is not a good preconditioner. This issue leads us to the derivation of the Modified Tangential Frequency Filtering Decomposition (MTFFD) preconditioner, in which the recursion formula of TFFD is modified by adding a term $c \Lambda_{i} h^{q}$. This idea of modification comes from the MILU preconditioner 20, where an additional term of order $\mathcal{O}\left(h^{-2}\right)(c \neq 0)$ is added to the diagonal along with dropped fill-in. For problems arising from the discretizations of second-order elliptic partial differential equations, it is known 6. 20 that the modification is able to reduce the condition number of the preconditioned matrix by MILU from $\mathcal{O}\left(h^{-2}\right)(c=0)$ to $\mathcal{O}\left(h^{-1}\right)(c \neq 0)$. Using a two dimensional Poisson equation as a model problem, we perform the Fourier analysis of the MTFFD preconditioner. The optimal choice of $q$ and $c$ are determined by this analysis, which shows that $q=\frac{4}{3}$ and $c=\left(4 \pi^{2}\right)^{\frac{2}{3}}$ are the optimal choices as $h$ tends to 0 . The optimality of these parameters is illustrated by the numerical tests. When the optimal choice of modification order $q$ is used, the Fourier analysis reveals that the condition number of the preconditioned matrix is $\mathcal{O}\left(h^{-\frac{2}{3}}\right)$. This bound is better compared with other incomplete factorization type preconditioners (c.f. [15]). To compare the preconditioning effect of MTFFD with TFFD, we present tests on large sparse matrices arising from the discretization of PDEs with discontinuous coefficients. The results show that the MTFFD preconditioner is much more efficient, and MTFFD preconditioned GMRES needs less than half of the iteration numbers of TFFD preconditioned GMRES.

We use $\operatorname{ctrid}_{m}(\alpha, \beta, \gamma)$ and $\operatorname{circ}_{m}\left(\gamma_{1}, \ldots, \gamma_{m}\right)$ to denote the tridiagonal circulant matrix and circulant matrix of order $m$, i.e.

$\operatorname{ctrid}_{m}(\alpha, \beta, \gamma)=\left[\begin{array}{cccc}\beta & \gamma & & \alpha \\ \alpha & \ddots & \ddots & \\ & \ddots & \ddots & \gamma \\ \gamma & & \alpha & \beta\end{array}\right], \operatorname{circ}_{m}\left(\gamma_{1}, \ldots, \gamma_{m}\right)\left[\begin{array}{ccccc}\gamma_{1} & \gamma_{2} & \ldots & \gamma_{m-1} & \gamma_{m} \\ \gamma_{m} & \gamma_{1} & \gamma_{2} & \ldots & \gamma_{m-1} \\ \vdots & \ddots & \ddots & \ddots & \vdots \\ \gamma_{3} & \ldots & \gamma_{m} & \gamma_{1} & \gamma_{2} \\ \gamma_{2} & \gamma_{3} & \ldots & \gamma_{m} & \gamma_{1}\end{array}\right]$

We also use $\operatorname{trid}_{m}(\alpha, \beta, \gamma)$ and $\operatorname{Btrid}_{m}(L, T, U)$ to denote the $m \times m$ tridiagonal and $m k \times m k$ block tridiagonal matrix with each diagonal block of size $k \times k$ respectively, i.e.

$\operatorname{trid}_{m}(\alpha, \beta, \gamma)=\left[\begin{array}{cccc}\beta & \gamma & & \\ \alpha & \ddots & \ddots & \\ & \ddots & \ddots & \gamma \\ & & \alpha & \beta\end{array}\right]$ and $\operatorname{Btrid}_{m}(L, T, U)=\left[\begin{array}{cccc}T & U & & \\ L & \ddots & \ddots & \\ & \ddots & \ddots & U \\ & & L & T\end{array}\right]$. 
The paper is organized as follows, In Section 2, a model problem is described, which will be used for Fourier analysis. In Section 3, we present the modified TFFD preconditioner and carry out the Fourier analysis for the MTFFD preconditioner. In Section 4, the performance of the MTFFD preconditioner is compared with the TFFD preconditioner by several examples. Finally, we conclude the paper in Section 5.

\section{Description of the model problem}

We consider the 2-D Poisson equation as the model problem, i.e.

$$
-\Delta u=f
$$

posed on the unit square $\Omega=0 \leq x, y \leq 1$ with Dirichlet boundary conditions

$$
u(x, y)=0 .
$$

This model problem is also used in [8, 15, 17. Discretizing this problem by the standard second-order finite difference (FE) scheme on a uniform grid with step size $h_{d}=\frac{1}{m+1}$ in each direction, we can obtain a linear system of order $m^{2}$

$$
\tilde{A} u=\tilde{b}
$$

where

$$
\begin{gathered}
\tilde{A}=I_{m} \otimes \tilde{D}+\kappa_{2} \tilde{S} \otimes I_{m}, \\
\tilde{D}=\operatorname{trid}_{m}\left(-\kappa_{1}, d,-\kappa_{1}\right), \\
\tilde{S}=\operatorname{trid}_{m}(-1,0,-1) .
\end{gathered}
$$

and $d=2\left(\kappa_{1}+\kappa_{2}\right), \kappa_{1}=\kappa_{2}=1$.

Fourier analysis can only be performed on constant coefficient problems with periodic boundary conditions [15]; hence we also introduce the discretization of equation (2) with periodic boundary conditions

$$
u(x, 0)=u(x, 1) \quad u(0, y)=u(1, y) .
$$

According to the argument in [15, we assume the discretization step size $h_{p}$ for the periodic case to be half that of Dirichlet case, i.e. $h_{p}=\frac{1}{n+1}=\frac{1}{2(m+1)}$. Then we have a linear system of order $n^{2}$

$$
A u=b
$$

where

$$
\begin{gathered}
A=I_{n} \otimes D+\kappa_{2} S \otimes I_{n}, \\
D=\operatorname{ctrid}_{n}\left(-\kappa_{1}, d,-\kappa_{1}\right), \\
S=\operatorname{ctrid}_{n}(-1,0,-1),
\end{gathered}
$$

and the values of $d, \kappa_{1}$ and $\kappa_{2}$ are the same as in (3).

The main idea of Fourier analysis to use the theoretical results obtained for a periodic problem to predict the convergence results of the corresponding 
Dirichlet problem. Therefore, we present in this paper the Fourier analysis of the linear system (4). In the following discussion, the equation (3) and (4) will be referred to as Dirichlet problem and periodic problem respectively, and we always use subscript $d$ and $p$ to distinguish the parameters for the Dirichlet case and the periodic case.

Firstly, the Fourier eigenvalue of the coefficient matrix $A$ is given by the following equation [15]

$$
A u^{(j, k)}=\lambda_{A} u^{(j, k)},
$$

where $u^{(j, k)}$ is defined by

$$
u_{s, t}^{(j, k)}=e^{i s \theta_{j}} e^{i t \phi_{k}}
$$

with

$$
\theta_{j}=\frac{2 \pi j}{n+1}, \quad \phi_{k}=\frac{2 \pi k}{n+1}, \quad 1 \leq j, k \leq n .
$$

and $i$ is the imaginary unit.

Substituting the expression of $u_{s, t}^{(j, k)}$ into the grid-equation related to $A$ (refer to [15]), we have

$$
\begin{aligned}
A u^{(j, k)} & =d u_{s, t}-\kappa_{1} u_{s+1, t}-\kappa_{1} u_{s-1, t}-\kappa_{2} u_{s, t-1}-\kappa_{2} u_{s, t+1} \\
& =\left(d-\kappa_{1} e^{\mathrm{i} \theta_{j}}-\kappa_{1} e^{-\mathrm{i} \theta_{j}}-\kappa_{2} e^{\mathrm{i} \phi_{k}}-\kappa_{2} e^{-\mathrm{i} \phi_{k}}\right) e^{s i \theta_{j}} e^{\mathrm{i} t \phi_{k}} \\
& =\left(4-2 \cos \left(\theta_{j}\right)-2 \cos \left(\phi_{k}\right)\right) e^{i s \theta_{j}} e^{\mathrm{i} t \phi_{k}} \\
& =4\left(\kappa_{1} \sin ^{2}\left(\frac{\theta_{j}}{2}\right)+\kappa_{2} \sin ^{2}\left(\frac{\phi_{k}}{2}\right)\right) u^{(j, k)}
\end{aligned}
$$

Thus, the Fourier eigenvalue of $A$ corresponding to the Fourier eigenvector $u^{(j, k)}$ are

$$
\lambda_{A}=\lambda_{j, k}(A)=4\left(\kappa_{1} \sin ^{2}\left(\frac{\theta_{j}}{2}\right)+\kappa_{2} \sin ^{2}\left(\frac{\phi_{k}}{2}\right)\right) .
$$

The expression of the Fourier eigenvalue of $A$ will be used later in the Fourier analysis.

\section{Modified Tangential Frequency Filtering De- compositions and its Fourier analysis}

For a general block tridiagonal linear system (3), we introduce the Modified Tangential Frequency Filtering Decomposition (MTFFD) preconditioner $\tilde{M}$ as follows

$\tilde{M}=\left[\begin{array}{cccc}\tilde{T}_{1} & & & \\ L_{1} & \tilde{T}_{2} & & \\ & \ddots & \ddots & \\ & & L_{m-1} & \tilde{T}_{m}\end{array}\right]\left[\begin{array}{cccc}\tilde{T}_{1}^{-1} & & & \\ & \tilde{T}_{2}^{-1} & & \\ & & \ddots & \\ & & & \tilde{T}_{m}^{-1}\end{array}\right]\left[\begin{array}{cccc}\tilde{T}_{1} & U_{1} & & \\ & \tilde{T}_{2} & \ddots & \\ & & \ddots & U_{m-1} \\ & & & \tilde{T}_{m}\end{array}\right]$

The diagonal blocks $\tilde{T}_{i}$ in MTFFD are computed by the following recursion formula

$$
\tilde{T}_{i}=\left\{\begin{array}{lr}
D_{1}+c \Lambda_{1} h^{q}, & i=1, \\
D_{i}-L_{i-1}\left(2 \beta_{i}-\beta_{i} \tilde{T}_{i-1} \beta_{i}\right) U_{i-1}+c \Lambda_{i} h^{q}, & 1<i \leq m .
\end{array}\right.
$$


where $\Lambda_{i}, 1 \leq i \leq m$, are diagonal matrices, parameter $q$ is the order of modification, and $c$ is a relaxation parameter. The optimal choice of $q$ and $c$ will be discussed later. The matrix $\beta_{i}$ is an approximation to the inverse of $\tilde{T}_{i-1}$, and it can be determined by enabling $\tilde{M}$ to have a filtering condition. The analysis in [2] shows that it reduce to solving

$$
\beta_{i}\left(U_{i-1} f\right)=\tilde{T}_{i-1}^{-1} U_{i-1} f,
$$

where $f$ is a filtering vector.

We can see that the MTFFD preoconditioner differs from the TFFD preconditioner in that an additional term $c \Lambda_{i} h^{q}$ is added in the recursion formula (10). If $\Lambda_{i}=I_{m}$, then the modification is similar to those done in the modified ILU factorization 6, 20, where the modification is $c h^{2} I_{m}$. The modification in (10) is quite similar to the shifted iteration methods discussed in 43, where the ILU factorization of a shifted coefficient matrix is constructed and used as a preconditioner for the original problem. For analysis purpose, we will fix $\Lambda_{i}=I_{m}, 1 \leq i \leq m$, and the filtering vector is chosen as $\mathbf{1}=[1, \ldots, 1]^{T}$.

As mentioned before, Fourier analysis can be performed only on the constant coefficient problems with periodic boundary conditions. According to the theory developed in [15], there are several assumptions on which our analysis will be based,

- The grid size $h_{p}=\frac{1}{2} h_{d}$ should be used in order to relate the Fourier analysis results to that of the Dirichlet problems. We have made this assumption be satisfied when the discretization of (2) is done.

- For the linear system (4) generated by the discretization of (2) with periodic boundary conditions, the MTFFD preconditioner $\hat{M}$ is forced to have constant diagonals, i.e. the MTFFD preconditioner $\hat{M}$ for periodic system (4) should take the form of

$$
\hat{M}=(L+\hat{T}) \hat{T}^{-1}(\hat{T}+U),
$$

where $\hat{T}$ has the same diagonal blocks, i.e.

$$
\hat{T}=I_{n} \otimes \hat{T}_{0}
$$

and each diagonal block $\hat{T}_{0}$ is circulant, i.e.

$$
\hat{T}_{0}=\operatorname{circ}_{n}\left(\hat{d},-\hat{\kappa}_{1}, 0, \ldots, 0,-\hat{\kappa}_{1}\right)
$$

with parameters $\hat{d}$ and $\hat{\kappa}_{1}$ to be determined by the recursion formula (10).

Using the assumptions above and the recursion formula (10), we now construct MTFFD preconditioner for which we will perform Fourier analysis. 
Firstly, the parameters $\hat{d}$ and $\hat{\kappa}_{1}$ can be computed by solving

$$
\begin{aligned}
\hat{T}_{i}= & D_{i}-L_{i-1}\left(2 \beta_{i-1}-\beta_{i-1} \hat{T}_{i-1} \beta_{i-1}\right) U_{i-1}+c h^{q} I_{n} \\
= & {\left[\begin{array}{cccc}
d & -\kappa_{1} & & -\kappa_{1} \\
-\kappa_{1} & d & \ddots & \\
& \ddots & \ddots & -\kappa_{1} \\
-\kappa_{1} & -\kappa_{1} & d
\end{array}\right]+\frac{\kappa_{2}^{2}}{\left(\hat{d}-2 \hat{\kappa}_{1}\right)^{2}}\left[\begin{array}{cccc}
\hat{d} & -\hat{\kappa}_{1} & & -\hat{\kappa}_{1} \\
-\hat{\kappa}_{1} & \hat{d} & \ddots & \\
& \ddots & \ddots & -\hat{\kappa}_{1} \\
-\hat{\kappa}_{1} & & -\hat{\kappa}_{1} & \hat{d}
\end{array}\right]-\frac{2 \kappa_{2}^{2}}{\left(\hat{d}-2 \hat{\kappa}_{1}\right)} I_{n}+\operatorname{ch}^{q} I_{n} } \\
= & {\left[\begin{array}{cccc}
d-\frac{\kappa_{2}^{2} \hat{d}-2 \kappa_{2}^{2}\left(\hat{d}-2 \hat{\kappa}_{1}\right)^{2}}{\left(\hat{d}-2 \hat{\kappa}_{1}\right)} & -\kappa_{1}-\frac{\kappa_{2}^{2} \hat{\kappa}_{1}}{\left(\hat{d}-\hat{\kappa}_{1}\right)^{2}} & & -\kappa_{1}-\frac{\kappa_{2}^{2} \hat{\kappa}_{1}}{\left(\hat{d}-2 \hat{\kappa}_{1}\right)^{2}} \\
-\kappa_{1}-\frac{\kappa_{2}^{2} \hat{\kappa}_{1}}{\left(\hat{d}-2 \hat{\kappa}_{1}\right)^{2}} & d-\frac{\kappa_{2}^{2} \hat{d}-2 \kappa_{2}^{2}\left(\hat{d}-2 \hat{\kappa}_{1}\right)^{2}}{\left(\hat{d}-2 \hat{\kappa}_{1}\right)} & \ddots & \\
-\kappa_{1}-\frac{\kappa_{2}^{2} \hat{\kappa}_{1}}{\left(\hat{d}-2 \hat{\kappa}_{1}\right)^{2}} & \ddots & \ddots & -\kappa_{1}-\frac{\kappa_{2}^{2} \hat{\kappa}_{1}}{\left(\hat{d}-2 \hat{\kappa}_{1}\right)^{2}} \\
- & & \kappa_{1}-\frac{\kappa_{2}^{2} \hat{\kappa}_{1}}{\left(\hat{d}-2 \hat{\kappa}_{1}\right)^{2}} & d-\frac{\kappa_{2}^{2} \hat{d}-2 \kappa_{2}^{2}\left(\hat{d}-2 \hat{\kappa}_{1}\right)^{2}}{\left(\hat{d}-2 \hat{\kappa}_{1}\right)}
\end{array}\right]+\operatorname{ch}^{q} I_{n} . }
\end{aligned}
$$

From the above relationship, we have

$$
\begin{gathered}
\hat{d}=d-\frac{\kappa_{2}^{2} \hat{d}-2 \kappa_{2}^{2}\left(\hat{d}-2 \hat{\kappa}_{1}\right)^{2}}{\left(\hat{d}-2 \hat{\kappa}_{1}\right)}+c h^{q}, \\
\hat{\kappa}_{1}=\kappa_{1}+\frac{\kappa_{2}^{2} \hat{\kappa}_{1}}{\left(\hat{d}-2 \hat{\kappa}_{1}\right)^{2}},
\end{gathered}
$$

or

$$
\begin{gathered}
(\hat{d}-d)\left(\hat{d}-2 \hat{\kappa}_{1}\right)^{2}=\kappa_{2}^{2}\left(\hat{d}-2 \hat{\kappa}_{1}\right)-2 \kappa_{2}^{2}\left(\hat{d}-2 \hat{\kappa}_{1}\right)+2 \hat{\kappa}_{1} \kappa_{2}^{2}, \\
\left(\hat{\kappa}_{1}-\kappa_{1}\right)\left(\hat{d}-2 \hat{\kappa}_{1}\right)^{2}=\kappa_{2}^{2} \hat{\kappa}_{1} .
\end{gathered}
$$

By using matlab symbolic computation [27, we have

$$
\begin{aligned}
\hat{d}-2 \hat{\kappa}_{1} & =-1+\frac{1}{2}\left(d+c h^{q}\right)+\frac{1}{2} \sqrt{(d+c h)^{2}-4\left(d+c h^{q}\right)} \\
& =1+\frac{1}{2} c h^{q}+\frac{1}{2} \sqrt{\left(4+c h^{q}\right) c h^{q}} \\
& =1+\eta_{h},
\end{aligned}
$$

and

$$
\begin{aligned}
\hat{\kappa}_{1} & =\frac{-\left(d+c h^{q}\right)-\sqrt{-4\left(d+c h^{q}\right)+\left(d+c h^{q}\right)^{2}}+\left(-1+\frac{1}{2}\left(d+c h^{q}\right)+\frac{1}{2} \sqrt{-4\left(d+c h^{q}\right)+\left(d+c h^{q}\right)^{2}}\right)\left(d+c h^{q}\right)}{\left(d+c h^{q}\right)\left(d+c h^{q}-4\right)} \\
& =\frac{1}{2}+\frac{\sqrt{\left(d+c h^{q}\right) c h^{q}}\left(\frac{1}{2}\left(d+c h^{q}\right)-1\right)}{c h^{q}\left(d+c h^{q}\right)} \\
& =\frac{1}{2}+\frac{\frac{1}{2} c h^{4}+1}{\sqrt{\left(4+c h^{q}\right) c h^{q}}} \\
& =\frac{1}{2}+\frac{1}{2 \delta_{h}},
\end{aligned}
$$

where $\eta_{h}=\frac{1}{2} c h^{q}+\frac{1}{2} \sqrt{\left(4+c h^{q}\right) c h^{q}}, \delta_{h}=\frac{\sqrt{\left(4+c h^{q}\right) c h^{q}}}{c h^{q}+2}$.

From (16) and (17) we can see that $\hat{\kappa}_{1} \stackrel{c h^{q}+2}{\rightarrow}$ as $c \rightarrow 0$. Thus, Fourier analysis can not be performed on the original tangential frequency filtering decomposition preconditioner.

By straightforward computation as in (7), the Fourier eigenvalues of $L, U$, and $\hat{T}$ are

$$
\begin{gathered}
\lambda_{L}=-\kappa_{2} e^{-\mathrm{i} \phi_{k}}, \\
\lambda_{U}=-\kappa_{2} e^{\mathrm{i} \phi_{k}},
\end{gathered}
$$




$$
\lambda_{\hat{T}}=\hat{d}-\hat{\kappa}_{1} \cos \left(\theta_{j}\right),
$$

respectively. Therefore, the Fourier eigenvalues of the MTFFD preconditioner $\hat{M}$ are

$$
\begin{aligned}
\lambda(\hat{M}) & =\left(\lambda_{L}+\lambda_{\hat{T}}\right) \lambda_{\hat{T}}^{-1}\left(\lambda_{U}+\lambda_{\hat{T}}\right) \\
& =\frac{\left(\hat{d}-2 \hat{\kappa}_{1} \cos \left(\theta_{j}\right)-\kappa_{2} \cos \left(\phi_{k}\right)+\mathrm{i} \kappa_{2} \sin \left(\phi_{k}\right)\right)\left(\hat{d}-2 \hat{\kappa}_{1} \cos \left(\theta_{j}\right)-\kappa_{2} \cos \left(\phi_{k}\right)-\mathrm{i} \kappa_{2} \sin \left(\phi_{k}\right)\right)}{\hat{d}-2 \hat{\kappa}_{1} \cos \left(\theta_{j}\right)} .
\end{aligned}
$$

Letting $\xi=\hat{d}-2 \hat{\kappa}_{1} \cos \left(\theta_{j}\right)$, we have

$$
\begin{aligned}
\lambda(\hat{M}) & =\frac{1}{\xi}\left(\xi-\kappa_{2} \cos \left(\phi_{k}\right)+i \kappa \sin \left(\phi_{k}\right)\left(\xi-\kappa_{2} \cos \left(\phi_{k}\right)-i \kappa_{2} \sin \left(\phi_{k}\right)\right)\right. \\
& =\frac{1}{\xi}\left(\left(\xi-\kappa_{2} \cos \left(\phi_{k}\right)\right)^{2}+\kappa_{1}^{2} \sin \left(\phi_{k}\right)^{2}\right) \\
& =\frac{1}{\xi}\left(\left(\xi^{2}-2 \xi \kappa_{2} \cos \left(\phi_{k}\right)+\kappa_{2}^{2}\right)\right.
\end{aligned}
$$

As we know,

Hence

$$
\begin{aligned}
\lambda\left(\hat{M}^{-1} A\right) & =\frac{4 \xi\left(\kappa_{1} \sin ^{2}\left(\frac{\theta_{j}}{2}\right)+\kappa_{2} \sin ^{2}\left(\frac{\phi_{k}}{2}\right)\right)}{\xi^{2}-2 \xi \kappa_{2} \cos \left(\phi_{k}\right)+\kappa_{2}^{2}} \\
& =\frac{4\left(\hat{d}-2 \hat{\kappa}_{1} \cos \left(\theta_{j}\right)\right)\left(\kappa_{1} \sin ^{2}\left(\frac{\theta_{j}}{2}\right)+\kappa_{2} \sin ^{2}\left(\frac{\phi_{k}}{2}\right)\right)}{\left(\hat{d}-2 \hat{\kappa}_{1} \cos \left(\theta_{j}\right)-\kappa_{2}\right)^{2}+2 \kappa_{2}\left(\hat{\theta}-2 \hat{\kappa}_{1} \cos \left(\theta_{j}\right)\right)\left(1-\cos \left(\phi_{k}\right)\right)} \\
& =\frac{4\left(\hat{d}-2 \hat{\kappa}_{1}+4 \hat{\kappa}_{1} \sin ^{2}\left(\frac{\theta_{j}}{2}\right)\right)\left(\kappa_{1} \sin ^{2}\left(\frac{\left(\frac{j}{2}\right.}{2}\right)+\kappa_{2} \sin ^{2}\left(\frac{\phi_{k}}{2}\right)\right)}{\left(\hat{d}-2 \hat{\kappa}_{1}+4 \hat{\kappa}_{1} \sin ^{2}\left(\frac{\theta_{j}}{2}\right)-\kappa_{2}\right)^{2}+4 \kappa_{2}\left(\hat{d}-2 \hat{\kappa}_{1}+4 \hat{\kappa}_{1} \sin ^{2}\left(\frac{\theta_{j}}{2}\right)\right) \sin ^{2}\left(\frac{\phi_{k}}{2}\right)} \\
& =\frac{4\left(\eta_{h}+1+4 \hat{\kappa}_{1} \sin ^{2}\left(\frac{\theta_{j}}{2}\right)\right)\left(\sin ^{2}\left(\frac{\theta_{j}}{2}\right)+\sin ^{2}\left(\frac{\phi_{k}}{2}\right)\right)}{\left(\eta_{h}+4 \hat{\kappa}_{1} \sin ^{2}\left(\frac{\theta_{j}}{2}\right)\right)^{2}+4 \kappa_{2}\left(\eta_{h}+1+4 \hat{\kappa}_{1} \sin ^{2}\left(\frac{\theta_{j}}{2}\right)\right) \sin ^{2}\left(\frac{\phi_{k}}{2}\right)} .
\end{aligned}
$$

Thus,

$$
\begin{aligned}
\lambda^{-1}\left(\hat{M}^{-1} A\right) & =\frac{\left(\eta_{h}+4 \hat{\kappa}_{1} \sin ^{2}\left(\frac{\theta_{j}}{2}\right)\right)^{2}+4 \kappa_{2}\left(\eta_{h}+1+4 \hat{\kappa}_{1} \sin ^{2}\left(\frac{\theta_{j}}{2}\right)\right) \sin ^{2}\left(\frac{\phi_{k}}{2}\right)}{4\left(\eta_{h}+1+4 \hat{\kappa}_{1} \sin ^{2}\left(\frac{\theta_{j}}{2}\right)\right)\left(\sin ^{2}\left(\frac{\theta_{j}}{2}\right)+\sin ^{2}\left(\frac{\phi_{k}}{2}\right)\right)} \\
& =\frac{16 \hat{\kappa}_{1}^{2} \sin ^{4}\left(\frac{\theta_{j}}{2}\right)+166 \hat{\kappa}_{1} \sin ^{2}\left(\frac{\theta_{j}}{2}\right) \sin ^{2}\left(\frac{\phi_{k}}{2}\right)+\eta_{h}^{2}+8 \delta_{h} \hat{\kappa}_{1} \sin ^{2}\left(\frac{\theta_{j}}{2}\right)+4\left(1+\eta_{h}\right) \sin ^{2}\left(\frac{\phi_{k}}{2}\right)}{16 \hat{\kappa}_{1} \sin ^{4}\left(\frac{\theta j_{j}}{2}\right)+16 \hat{\kappa}_{1} \sin ^{2}\left(\frac{\theta_{j}}{2}\right) \sin ^{2}\left(\frac{\phi_{h}}{2}\right)+4\left(1+\eta_{h}\right)\left(\sin ^{2}\left(\frac{\theta_{j}}{2}\right)+\sin ^{2}\left(\frac{\phi_{h}}{2}\right)\right)} \\
& =1+\frac{16\left(\hat{\kappa}_{1}-1\right) \hat{\kappa}_{1} \sin ^{4}\left(\frac{\theta_{j}}{4}\right)+\eta_{h}^{2}+8 \hat{\kappa}_{1} \eta_{h} \sin ^{2}\left(\frac{\theta_{j}}{2}\right)-4\left(1+\eta_{h}\right) \sin ^{2}\left(\frac{\theta_{j}}{2}\right)}{16 \hat{\kappa}_{1} \sin ^{4}\left(\frac{\theta_{j}}{2}\right)+16 \hat{\kappa}_{1} \sin ^{2}\left(\frac{\theta_{j}}{2}\right) \sin ^{2}\left(\frac{\phi_{k}}{2}\right)+4\left(1+\eta_{h}\right)\left(\sin ^{2}\left(\frac{\theta_{j}}{2}\right)+\sin ^{2}\left(\frac{\phi_{k}}{2}\right)\right)} \\
& =1+\frac{4\left(\delta_{h}^{-1}+1\right)\left(\delta_{h}^{-1}-1\right) \sin ^{2}\left(\frac{\theta_{j}}{2}\right)+\frac{\eta_{h}^{2}}{\sin ^{2}\left(\frac{\theta_{j}}{2}\right)}}{16 \hat{\kappa}_{1} \sin ^{2}\left(\frac{\theta_{j}}{2}\right)+16 \hat{\kappa}_{1} \sin ^{2}\left(\frac{\phi_{k}}{2}\right)+4\left(1+\eta_{h}\right)+4\left(1+\eta_{h}\right) \frac{\sin ^{2}\left(\frac{\phi_{h}}{2}\right)}{\sin ^{2}\left(\frac{j_{j}}{2}\right)}} .
\end{aligned}
$$

As $h$ tends to 0 , we have $\delta_{h}^{-1} \geq 1$. Then from (21) it is easy to see that asymptotically

$$
\lambda^{-1}\left(\hat{M}^{-1} A\right) \geq 1,
$$

i.e. $\lambda\left(\hat{M}^{-1} A\right) \leq 1$. This is consistent with the theoretical results obtained in [2].

Subsequently, we will derive the upper bound of $\lambda^{-1}\left(\hat{M}^{-1} A\right)$ in an analytical way.

Let

$$
f\left(s_{1}, s_{2}\right)=1+\frac{\alpha s_{1}^{2}+\frac{\gamma}{s_{1}^{2}}}{\beta s_{1}^{2}+\beta s_{2}^{2}+e \frac{s_{2}^{2}}{s_{1}^{2}}+e},
$$


where we use $s_{1}=\sin \left(\frac{\theta}{2}\right), s_{2}=\sin \left(\frac{\phi}{2}\right)$. It is easy to see that $f\left(s_{1}, s_{2}\right)$ is a continuous function of $\sin \left(\frac{\theta}{2}\right)$ and $\sin \left(\frac{\phi}{2}\right)$, with $\left(\frac{\theta}{2}, \frac{\phi}{2}\right)$ defined in $(0, \pi) \times(0, \pi)$. In the representation form of $f\left(s_{1}, s_{2}\right)$, we have set $\alpha=4\left(\delta_{h}^{-1}+1\right)\left(\delta_{h}^{-1}-1\right)$, $\gamma=\eta_{h}^{2}, \beta=16 \hat{\kappa}_{1}$, and $e=4\left(1+\eta_{h}\right)$.

Taking partial derivation of $f\left(s_{1}, s_{2}\right)$ with $s_{2}$, we have

$$
f_{s_{2}}^{\prime}\left(s_{1}, s_{2}\right)=\frac{-2\left(\alpha s_{1}^{2}+\frac{\gamma}{s_{1}^{2}}\right)\left(\beta+\frac{e}{s_{1}^{2}}\right) s_{2} c_{2}}{\left(\beta s_{1}^{2}+\beta s_{2}^{2}+e \frac{s_{2}^{2}}{s_{1}^{2}}+e\right)^{2}} \begin{cases}\leq 0, & \phi \in\left(0, \frac{\pi}{2}\right), \\ >0, & \phi \in\left(\frac{\pi}{2}, \pi\right),\end{cases}
$$

where $c_{2}=\cos \left(\frac{\phi}{2}\right)$.

Thus,

$$
\max _{\theta, \phi}\left(f\left(s_{1}, s_{2}\right)\right)=f\left(s_{1}, 0\right)=f\left(s_{1}, \pi\right)=1+\frac{\alpha s_{1}^{2}+\frac{\gamma}{s_{1}^{2}}}{\beta s_{1}^{2}+e} .
$$

Therefore, the maximum value of $\lambda\left(\hat{M}^{-1} A\right)$ is attained on the line $(j, k)$ with $k=1$, or $k=n$.

Also we have

$$
\begin{aligned}
f_{s_{1}}^{\prime}\left(s_{1}, 0\right) & =\frac{4 \alpha s_{1}^{3} c_{1}\left(\beta s_{1}^{4}+e s_{1}^{2}\right)-\left(\alpha s_{1}^{4}+\gamma\right)\left(4 \beta s_{1}^{3} c_{1}+2 e s_{1} c_{1}\right)}{\left(\beta s_{1}^{4}+e s_{1}^{2}\right)^{2}} \\
& =\frac{2 \alpha e s_{1}^{4}-\gamma\left(4 \beta s_{1}^{2}+2 e\right)}{\left(\beta s_{1}^{4}+e s_{1}^{2}\right)^{2}} s_{1} c_{1} \\
& =\frac{32\left(\delta_{h}^{-2}-1\right)\left(1+\eta_{h}\right) s_{1}^{4}-\frac{32 \eta_{h}^{2}\left(1+\delta_{h}\right) s_{1}^{2}}{\delta_{h}}-8 \eta_{h}^{2}\left(1+\eta_{h}\right)}{\left(\beta s_{1}^{4}+e s^{2}\right)^{2}} s_{1} c_{1} \\
& \approx \frac{s_{1} c_{1}}{\left(\beta s_{1}^{4}+e s_{1}^{2}\right)^{2}}\left(\frac{32 s_{1}^{1}}{\delta_{h}^{2}}-\frac{32 \eta_{h}^{2} s_{1}^{2}}{\delta_{h}}\right) \\
& \approx \frac{s_{1} c_{1}}{\left(\beta s_{1}^{4}+e s_{1}^{2}\right)^{2}}\left(\frac{32 s_{1}^{4}}{c h^{q}}-32 \sqrt{c} h^{\frac{q}{2}} s_{1}^{2}\right) .
\end{aligned}
$$

In the above approximation, the high-order terms are ignored as $h$ is assumed to be sufficiently small. Subsequently, we will analyze the sign of $f_{s_{1}}^{\prime}\left(s_{1}, 0\right)$ in two cases:

- When $q \geq \frac{4}{3}$, then as $h \rightarrow 0$, we have

$$
f_{s_{1}}^{\prime}\left(s_{1}, 0\right) \quad \text { is } \begin{cases}\geq 0, & \theta \in\left(0, \frac{\pi}{2}\right] \\ <0, & \theta \in\left(\frac{\pi}{2}, \pi\right)\end{cases}
$$

Therefore, the maximum value of $\lambda^{-1}\left(\tilde{M}^{-1} A\right)$ is attained whenever $j=\left\lfloor\frac{n}{2}\right\rfloor+1$, and $k=1$ or $k=n$, where $\left\lfloor\frac{n}{2}\right\rfloor$ denotes the largest integer less than $\frac{n}{2}$. At these points $(j, k)$, we have

$$
\begin{aligned}
\lambda_{\left\lfloor\frac{n}{2}\right\rfloor+1, k}^{-1}\left(\tilde{M}^{-1} A\right) & \approx 1+\frac{\frac{4}{c h^{q}}+c h^{q}-4}{\frac{8}{\sqrt{c h^{q}}+4}} \\
& \approx 1+\frac{1}{2 \sqrt{c} h^{\frac{q}{2}}} \\
& \geq \frac{1}{2 \sqrt{c h} h^{\frac{2}{3}}}
\end{aligned}
$$

- When $0 \leq q \leq \frac{4}{3}$, then as $h \rightarrow 0$, we have

$$
f_{s_{1}}^{\prime}\left(s_{1}, 0\right) \quad \text { is } \quad \begin{cases}\leq 0, & \theta \in\left(0, \xi_{h}\right], \\ >0, & \theta \in\left(\xi_{h}, \frac{\pi}{2}\right), \\ \leq 0, & \theta \in\left[\frac{\pi}{2}, \pi-\xi_{h}\right], \\ >0, & \theta \in\left(\pi-\xi_{h}, \pi\right),\end{cases}
$$


where $\xi_{h}$ the positive angle such $\frac{32 s_{1}^{4}}{c h^{q}}=32 \sqrt{c} h^{\frac{q}{2}} s_{1}^{2}$, i.e. $s_{1}^{2}=c^{\frac{3}{2}} h^{\frac{3 q}{2}}$. From the equality, we have $\xi_{h}=\arcsin \left(c^{\frac{3}{4}} h^{\frac{3 q}{4}}\right)$. Therefore, in this case, the maximum value of $\lambda^{-1}\left(\hat{M}^{-1} A\right)$ is possibly attained at one of the following three points $(j, k)=(1, k),(j, k)=(n, k)$, or $(j, k)=\left(\left\lfloor\frac{n}{2}\right\rfloor+1, k\right)$, with $k=1$ or $k=n$. At the first two points, we have

$$
\begin{aligned}
\lambda_{1, k}^{-1}\left(\hat{M}^{-1} A\right)=\lambda_{n, k}^{-1}\left(\hat{M}^{-1} A\right) & \approx 1+\frac{c h_{p}^{q}}{8 \pi^{2} h^{2}} \\
& \approx 1+\frac{c}{8 \pi^{2} h^{2-q}} \\
& \geq \frac{c \pi^{2} h^{\frac{2}{3}}}{}
\end{aligned}
$$

At the third point, we have

$$
\begin{aligned}
\lambda_{\left\lfloor\frac{n}{2}\right\rfloor+1, k}^{-1}\left(\tilde{M}^{-1} A\right) & \approx 1+\frac{\frac{4}{c h}+c h^{q}-4}{\frac{8}{\sqrt{\operatorname{ch}^{q}}+4}} \\
& \approx 1+\frac{1}{2 \sqrt{c} h^{\frac{q}{2}}} \\
& \leq \frac{1}{2 \sqrt{c} h^{\frac{2}{3}}} .
\end{aligned}
$$

Therefore, in this case the maximum value of $\lambda^{-1}\left(\hat{M}^{-1} A\right)$ is attained at $(j, k)=$ $(1,1)$, or $(j, k)=(n, n)$, i.e. the value shown by (28).

As we have shown above, the maximum eigenvalue of the preconditioned matrix is approximately equal to 1 . Therefore, the condition number of the preconditioned matrix is approximately given by

$$
\begin{aligned}
\kappa\left(\hat{M}^{-1} A\right) & =\frac{\max _{j, k} \lambda_{j, k}\left(\hat{M}^{-1} A\right)}{\min _{j, k} \lambda_{j, k}\left(\hat{M}^{-1} A\right)} \\
& \approx \frac{\lambda_{\max }^{-1}\left(\hat{M}^{-1} A\right)}{1}
\end{aligned}
$$

where $\lambda_{\text {max }}^{-1}\left(\hat{M}^{-1} A\right)$ denotes the maximum value of $\lambda^{-1}\left(\hat{M}^{-1} A\right)$. For fixed $c$, from the above analysis we can see that the optimal $q$ that minimizes the condition number is attained at $\frac{4}{3}$.

Define

$$
\left\{\begin{array}{l}
g_{1}(c)=1+\frac{1}{2 \sqrt{c} h^{\frac{2}{3}}}, \\
g_{2}(c)=1+\frac{c}{8 \pi^{2} h^{\frac{2}{3}}} .
\end{array}\right.
$$

Then the optimal $c$ can be determined by solving

$$
\min _{c} \max \left\{g_{1}(c), g_{2}(c)\right\} \text {. }
$$

This min-max problem can be solved by their plot. In Figure 1 we give the curve of function $g_{1}(c)$ and $g_{2}(c)$ when $h^{\frac{2}{3}}=0.1$. From the figure we can see that (30) is solved whenever

$$
g_{1}(c)=g_{2}(c) .
$$

From this equation, we can get the optimal $c=\left(4 \pi^{2}\right)^{\frac{2}{3}}$.

Suppose $q$ is chosen as $\frac{4}{3}$, then the condition estimate of the preconditioned linear system is

$$
\begin{aligned}
\kappa\left(\hat{M}^{-1} A\right) & =\frac{\max _{j, k} \lambda_{j, k}\left(\hat{M}^{-1} A\right)}{\min _{j, k} \lambda_{j, k}\left(\hat{M}^{-1} A\right)} \\
& \approx \frac{\lambda_{\max }\left(\hat{M}^{-1} A\right)^{-1}}{1} \\
& \leq 1+\max \left\{\frac{1}{2 \sqrt{c} h^{\frac{2}{3}}}, \frac{c}{8 \pi^{2} h^{\frac{2}{3}}}\right\} \\
& =\mathcal{O}\left(h^{-\frac{2}{3}}\right) .
\end{aligned}
$$




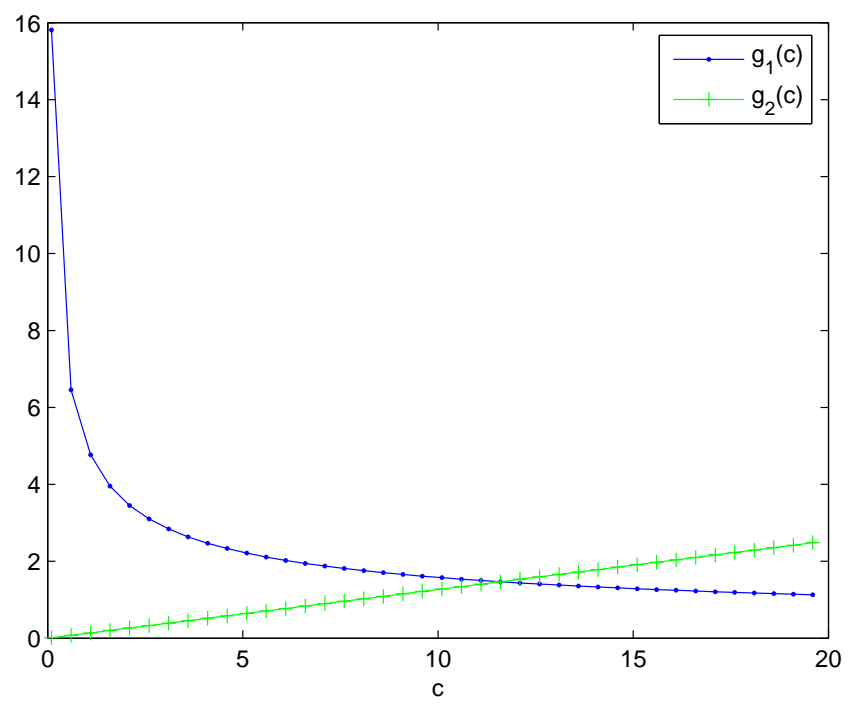

Figure 1: The curves of function $g_{1}(c)$ and $g_{2}(c)$.

We remark that the analysis above is for a periodic problem. If subscripts of $c_{d}$ and $c_{p}$ are used to distinguish the parameters for Dirichlet and periodic problems, then the optimal $c_{p}=\left(4 \pi^{2}\right)^{\frac{2}{3}}$. From the mesh size relationship of Dirichlet and periodic problems, we have $h_{p}=\frac{1}{2} h_{d}$. Hence, For $q=\frac{4}{3}$, the modification should satisfy the relationship [17]

$$
c_{p} h_{p}^{\frac{4}{3}}=c_{d} h_{d}^{\frac{4}{3}}
$$

from where we can get the optimal $c_{d}$, i.e. $c_{d}=\left(\frac{1}{2}\right)^{\frac{4}{3}}\left(4 \pi^{2}\right)^{\frac{2}{3}} \approx 4.6012$.

The above analysis can be concluded by the following theorem

Theorem 1 For the MTFFD preconditioner with $\Lambda_{i}$ be an identity matrix, the optimal choice of modification order is $q=\frac{4}{3}$, the optimal relaxation parameter is $c_{p}=\left(4 \pi^{2}\right)^{\frac{2}{3}}$. For $q=\frac{4}{3}$ and fixed $c$, then asymptotically $(h \rightarrow 0)$ the eigenvalues of MTFFD preconditioned matrix $\hat{M}^{-1} A$ are always less than 1 , and the condition number of $\hat{M}^{-1} A$ is $\mathcal{O}\left(h^{-\frac{2}{3}}\right)$.

Remarks: By using the semi-discrete analysis, Y. Achdou and F. Nataf (cf. Reference [1]) obtain an optimal filtering vector that minimizes the condition number of preconditioned matrix by the tangential frequency filtering preconditioner 38. In this paper, we choose $\mathbf{1}$ as the filtering vector, but modify the recursion formula of tangential tangential filtering decomposition proposed in 2. The optimal condition numbers obtained in both papers have the same order. In Reference [13, the same order of the condition number is obtained by using optimized two-frequency filtering decomposition. However, it is not tangential filtering decomposition. 


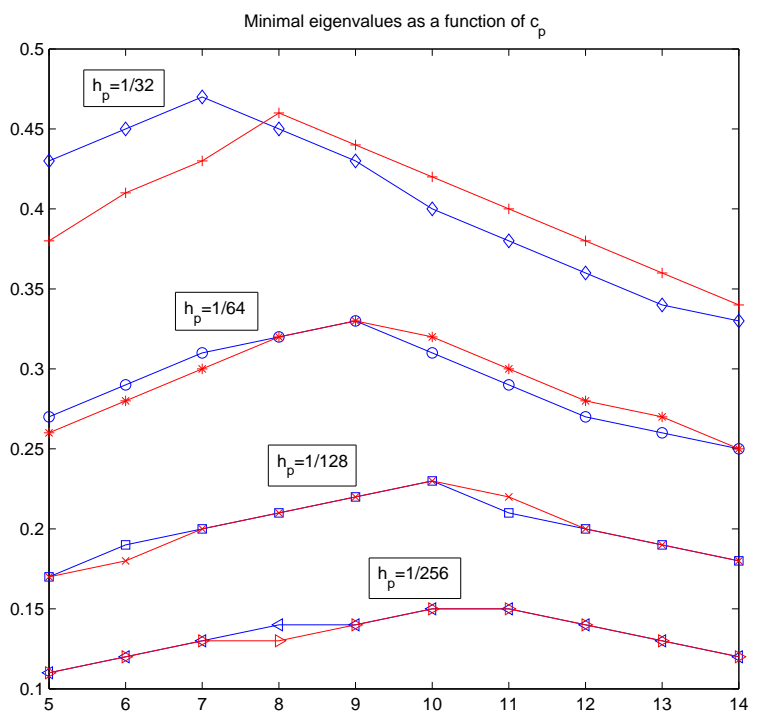

Figure 2: The dependence of minimum eigenvalues on parameter $c_{p}$.

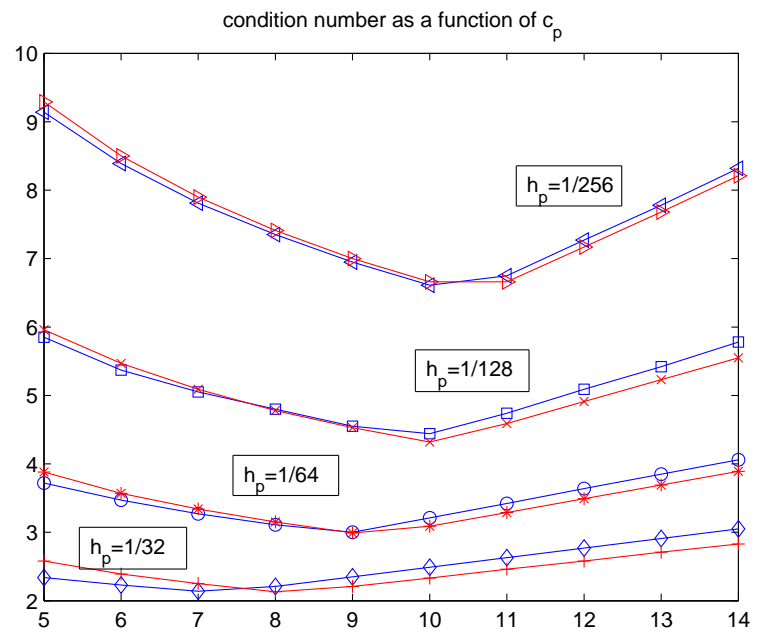

Figure 3: The dependence of condition numbers on parameter $c_{p}$ 
Table 1: Dirichlet and periodic results for $c_{d}=2.5$.

\begin{tabular}{|l|l|l|l|l|l|l|}
\hline$c_{d}=2.5$ & $\lambda_{\max }$ & \multicolumn{3}{|l|}{$\lambda_{\min }$} & \multicolumn{2}{l|}{ condition number } \\
\hline$\frac{1}{h_{d}}$ & Dirichlet & Periodic & Dirichlet & Periodic & Dirichlet & Periodic \\
\hline 8 & 1.00 & 0.97 & 0.64 & 0.54 & 1.55 & 1.77 \\
\hline 16 & 1.00 & 0.99 & 0.43 & 0.38 & 2.34 & 2.58 \\
\hline 32 & 1.00 & 1.00 & 0.27 & 0.26 & 3.72 & 3.88 \\
\hline 64 & 1.00 & 1.00 & 0.17 & 0.17 & 5.83 & 5.96 \\
\hline 128 & 1.00 & 1.00 & 0.11 & 0.11 & 9.14 & 9.28 \\
\hline 256 & 1.00 & 1.00 & 0.07 & 0.07 & 14.37 & 14.58 \\
\hline
\end{tabular}

Table 2: Dirichlet and periodic results for $c_{d}=5$.

\begin{tabular}{|l|l|l|l|l|l|l|}
\hline$c_{d}=5$ & $\lambda_{\max }$ & \multicolumn{2}{|l|}{$\lambda_{\min }$} & \multicolumn{2}{l|}{ condition number } \\
\hline$\frac{1}{h_{d}}$ & Dirichlet & Periodic & Dirichlet & Periodic & Dirichlet & Periodic \\
\hline 8 & 1.00 & 0.94 & 0.49 & 0.51 & 2.03 & 1.84 \\
\hline 16 & 1.00 & 0.98 & 0.40 & 0.42 & 2.49 & 2.33 \\
\hline 32 & 1.00 & 0.99 & 0.31 & 0.32 & 3.21 & 3.09 \\
\hline 64 & 1.00 & 1.00 & 0.23 & 0.23 & 4.40 & 4.32 \\
\hline 128 & 1.00 & 1.00 & 0.15 & 0.15 & 6.61 & 6.66 \\
\hline 256 & 1.00 & 1.00 & 0.097 & 0.096 & 10.32 & 10.39 \\
\hline
\end{tabular}

Table 3: Dirichlet and periodic results for $c_{d}=7.5$.

\begin{tabular}{|l|l|l|l|l|l|l|}
\hline$c_{d}=7.5$ & \multicolumn{2}{|l|}{$\lambda_{\max }$} & \multicolumn{2}{|l|}{$\lambda_{\min }$} & \multicolumn{2}{l|}{ condition number } \\
\hline$\frac{1}{h_{d}}$ & Dirichlet & Periodic & Dirichlet & Periodic & Dirichlet & Periodic \\
\hline 8 & 1.00 & 0.96 & 0.40 & 0.42 & 2.53 & 2.20 \\
\hline 16 & 1.00 & 0.96 & 0.31 & 0.33 & 3.20 & 2.95 \\
\hline 32 & 1.00 & 0.99 & 0.23 & 0.24 & 4.28 & 4.09 \\
\hline 64 & 1.00 & 0.99 & 0.17 & 0.17 & 6.02 & 5.88 \\
\hline 128 & 1.00 & 1.00 & 0.11 & 0.11 & 8.83 & 8.69 \\
\hline 256 & 1.00 & 1.00 & 0.075 & 0.076 & 13.27 & 13.21 \\
\hline
\end{tabular}

In Figures 2-3. the minimum eigenvalues and the condition numbers are plotted as a function of $c$, with $\frac{1}{16}, \frac{1}{32}, \frac{1}{64}, \frac{1}{128}$ (corresponding to $h_{p}=\frac{1}{32}, \frac{1}{64}, \frac{1}{128}, \frac{1}{256}$ ). As the maximum eigenvalues are both close to 1 and their plots are not easy to distinguish, so we don't display them. From the figures, it is easy to see that the minimal eigenvalue (and hence the condition numbers) are quite similar. From Figure 3. we can see that the experimental optimal $c_{p}$ is a little smaller than the theoretical asymptotical optimal value. This is possibly because the mesh size is not sufficiently refined. However, as $h_{p} \rightarrow 0$, the experimental optimal value $c$ indeed tends to $\left(4 \pi^{2}\right)^{\frac{2}{3}}$.

As we have shown above, the experimental optimal parameter $c_{p}$ is slightly smaller than the asymptotically optimal value $\left(4 \pi^{2}\right)^{\frac{2}{3}}=11.59$. In the following test, we compare the numerical results with three different parameter $c_{p}$ and various mesh sizes. Three parameters $c_{p}=5, c_{p}=10$, and $c_{p}=15$ are chosen; 


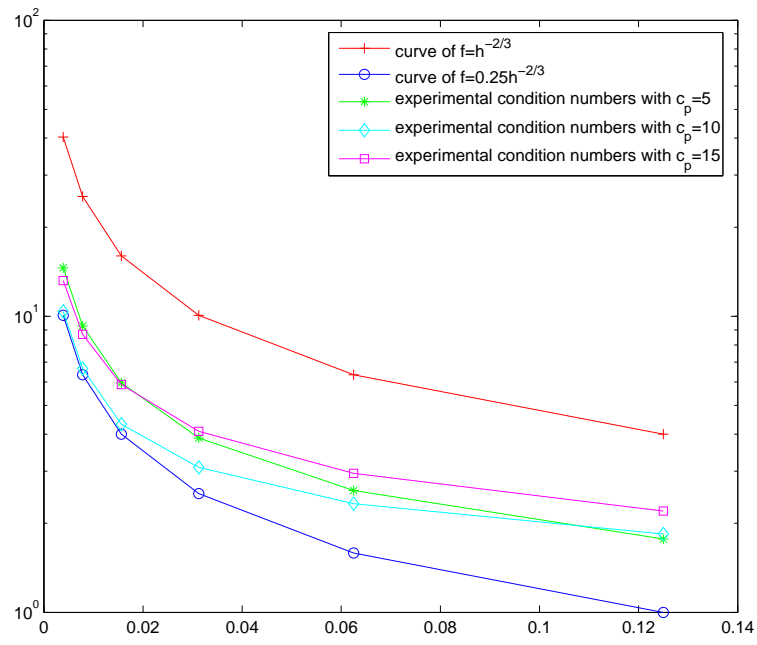

Figure 4: The dependence of condition numbers on $h_{p}$.

in the sense that $c_{p}=5$ is less than the optimal value; $c_{p}=10$ is close to the optimal value; and $c_{p}=15$ is larger than the optimal value. The test results are shown in Table 11-3. where we use Dirichlet and Periodic to denote the results for Dirichlet case and periodic case, respectively. In order to approximate the extremal eigenvalues of the preconditioned Dirichlet system, we use restarted harmonic Arnoldi method 31] when mesh size $h_{d} \leq \frac{1}{64}$. The computed approximate eigenpairs $\left(\lambda_{i}, \hat{\varphi}_{i}\right)$ satisfy $\left\|A \hat{\varphi}_{i}-\hat{\lambda}_{i} \hat{\varphi}_{i}\right\|<10^{-2}$. From the three tables, we can see that the periodic values are very close to the Dirichlet values. The condition number of the preconditioned Dirichlet system can be captured by the periodic results. By comparing Table 1 Table 3 with Table 2 respectively, we can see that $c_{d}=5$ produces the best condition number as $h_{d}$ is refined. The results are consistent with the theoretical results.

To illustrate that the condition number of $\hat{M}^{-1} A$ is $\mathcal{O}\left(h^{-\frac{2}{3}}\right)$, we display in Figure 4 the experimental periodical condition numbers of Tables 1 . 3. The $\mathrm{x}$-axis denotes the values of $h_{p}$; the y-axis is the logarithmic scale of the experimental condition numbers and the function values of $h_{p}^{-\frac{2}{3}}$ and $\frac{1}{4} h_{p}^{-\frac{2}{3}}$. The results at the points $h_{p}=\frac{1}{8}, \frac{1}{16}, \frac{1}{32}, \frac{1}{64}, \frac{1}{128}, \frac{1}{256}$ are plotted. From the Figures we can see that the periodical experimental condition numbers depend linearly on $h_{p}^{-\frac{2}{3}}$. As $h_{p}$ tends to zero, the plot of experimental results with $c_{p}=10$ (c.f. Table 2) becomes very close to the curve of function $\frac{1}{4} h_{p}^{-\frac{2}{3}}$.

To compare the range and clustering of the Fourier eigenvalues with that of preconditioned Dirichlet system, we display the spectrum distributions when $c_{p}=5$ and $c_{p}=10$, see Figures [5] and [6] respectively. The test results of mesh size $h_{d}=\frac{1}{8}, \frac{1}{16}, \frac{1}{32}$, (corresponding to $h_{p}=\frac{1}{16}, \frac{1}{32}, \frac{1}{64}$ ) are plotted. From the Figures, we see that the range and clustering of the preconditioned Dirichlet system and the Fourier eigenvalue distribution are extremely close. As mesh 

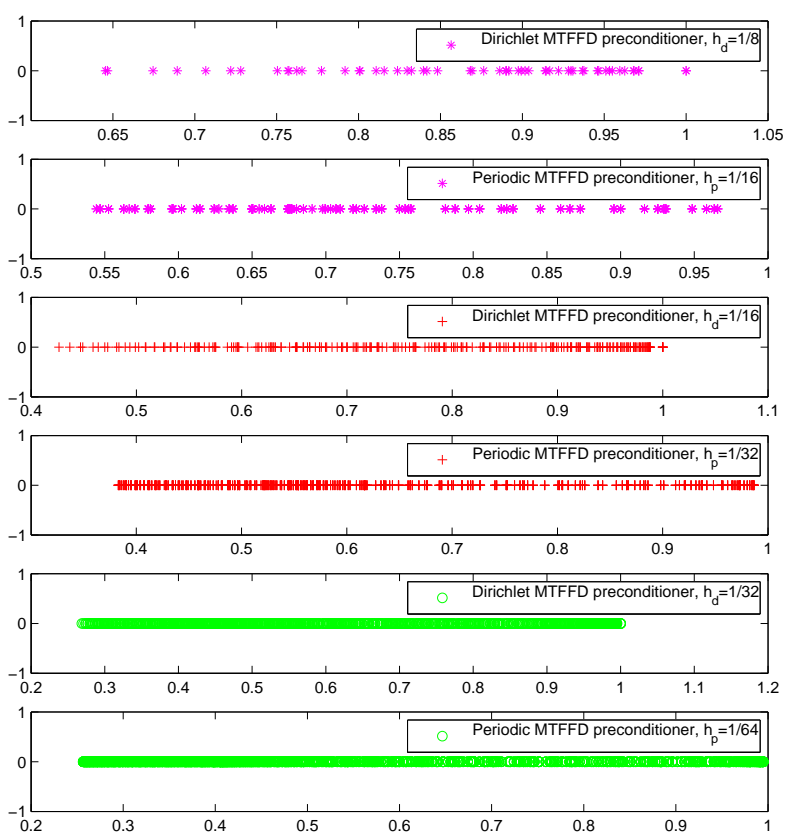

Figure 5: Spectrum distribution of the preconditioned matrices, with $c_{p}=5$. 


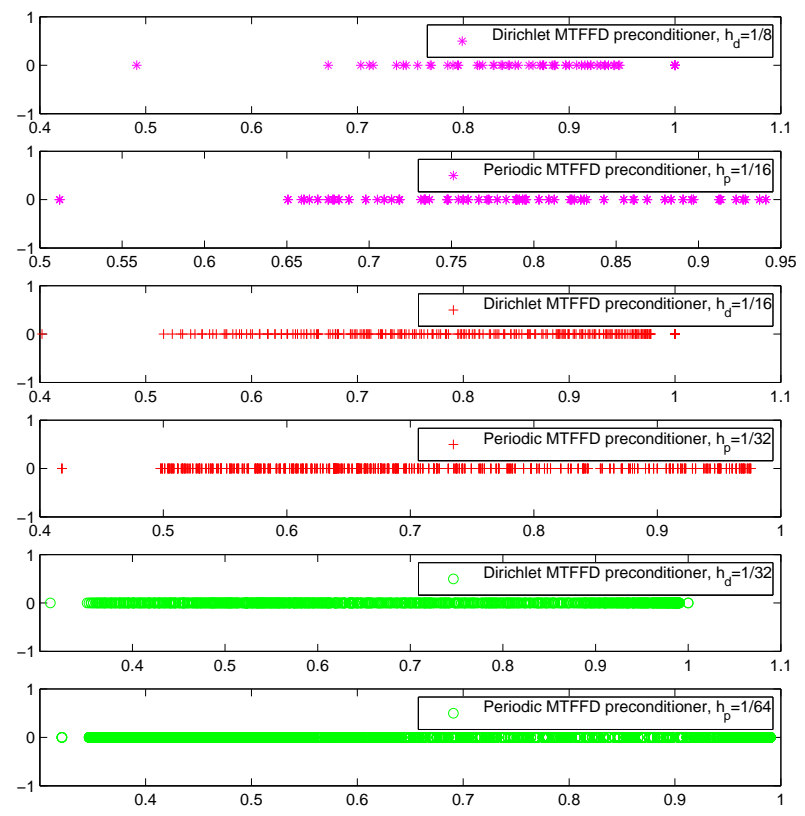

Figure 6: Spectrum distribution of the preconditioned matrices, with $c_{p}=10$.

size $h$ decreases, the extremal eigenvalues (and hence the condition number) of both cases become closer.

\section{Numerical Examples}

The performance of the MTFFD preconditioner, the TFFD preconditioner 2], and the ILU(0) 36 preconditioner are compared on several problems arising from the discretization of partial differential equations. All the tests are run on an Intel Pentium IV Dual-Core with main memory $1 \mathrm{G}$ and the machine precision eps $=2.22 \times 10^{-16}$ using MATLAB 7.5 on a Linux-based system.

In the tests, we stop the algorithm when the relative norm $\frac{\left\|b-A x_{k}\right\|}{\|b\|}$ is less than $10^{-12}$. Both the exact solution and the initial approximate solutions are chosen randomly. In the following discussions, the restarted GMRES [36] is used with maximum subspace dimension 200. The filtering vector is always chosen as $\mathbf{1}=[1, \ldots, 1]^{T}$. In the following tables, iter denotes the number of iterations, error denotes the infinite norm of the difference between the final approximate solution and the exact solution. We use "†" to denote that the method fails to converge within 200 iteration steps. 
Example 1. We consider the boundary value problem as in [2]

$$
\begin{array}{r}
\eta(x) u+\operatorname{div}(\mathbf{a}(x) u)-\operatorname{div}(\kappa(x) \nabla u)=f \text { in } \Omega \\
u=0 \text { on } \partial \Omega_{D} \\
\frac{\partial u}{\partial n}=0 \text { on } \partial \Omega_{N}
\end{array}
$$

where $\Omega=[0,1]^{k}$ with $k=2, \partial \Omega_{N}=\partial \Omega \backslash \partial \Omega_{D}, \partial \Omega_{D}=[0,1] \times\{0,1\}$.

We consider the following five different cases. As these problems are no longer constant coefficient, we choose the additional term as $c \Lambda_{i} h^{\frac{4}{3}}$, where $\Lambda_{i}=$ $\operatorname{diag}\left(D_{i}\right)$, i.e. the diagonal matrix of the $i$ th diagonal block of the coefficient matrix.

Case I: The advection-diffusion problem with a rotating velocity in two dimensions:

The tensor $\kappa$ is the identity, and the velocity is $\mathbf{a}(x)=\left(2 \pi\left(x_{2}-0.5\right), 2 \pi\left(x_{1}-\right.\right.$ $0.5))^{T}$. The function $\eta(x)$ is zero. The uniform grid with $n \times n$ nodes, $n=$ 100, 200,300, 400 nodes are tested respectively. The diagonal elements of $A$ are close to 4 . We set parameter $c$ to be 2.5 in the numerical test. Table 4 displays the results obtained by using three different preconditioners.

Table 4: Test results for advection-diffusion problems, nonsymmetric matrix

\begin{tabular}{|r|r|r|r|r|r|r|}
\hline & \multicolumn{2}{|c|}{ MTFFD } & \multicolumn{2}{c|}{ TFFD } & \multicolumn{2}{|c|}{ ILU(0) } \\
\hline $1 / h$ & iter & error & iter & error & iter & error \\
\hline 100 & 26 & $2.4 \mathrm{e}-13$ & 57 & $8.3 \mathrm{e}-12$ & 108 & $7.3 \mathrm{e}-10$ \\
\hline 200 & 32 & $5.6 \mathrm{e}-9$ & 82 & $1.3 \mathrm{e}-11$ & 191 & $3.2 \mathrm{e}-9$ \\
\hline 300 & 37 & $9.7 \mathrm{e}-10$ & 101 & $1.6 \mathrm{e}-11$ & $\dagger$ & $2.4 \mathrm{e}-6$ \\
\hline 400 & 40 & $8.1 \mathrm{e}-12$ & 117 & $1.4 \mathrm{e}-11$ & $\dagger$ & $2.0 \mathrm{e}-5$ \\
\hline
\end{tabular}

Table 5: Test results for non-Homogeneous problems, symmetric matrix

\begin{tabular}{|r|r|r|r|r|r|r|}
\hline & \multicolumn{2}{|c|}{ MTFFD } & \multicolumn{2}{|c|}{ TFFD } & \multicolumn{2}{|c|}{ ILU(0) } \\
\hline $1 / h$ & iter & error & iter & error & iter & error \\
\hline 100 & 26 & $8.7 \mathrm{e}-13$ & 57 & $7.5 \mathrm{e}-12$ & 108 & $3.0 \mathrm{e}-10$ \\
\hline 200 & 32 & $3.0 \mathrm{e}-12$ & 82 & $9.2 \mathrm{e}-12$ & 186 & $4.3 \mathrm{e}-9$ \\
\hline 300 & 37 & $5.8 \mathrm{e}-10$ & 101 & $1.4 \mathrm{e}-11$ & $\dagger$ & $2.6 \mathrm{e}-6$ \\
\hline 400 & 41 & $1.1 \mathrm{e}-12$ & 116 & $1.7 \mathrm{e}-11$ & $\dagger$ & $4.9 \mathrm{e}-5$ \\
\hline
\end{tabular}

Case II: Non-Homogenous problems with large jumps in the coefficients in two dimensions:

The coefficient $\eta(x)$ and $\mathbf{a}(x)$ are both zero. The tensor $\kappa$ is isotropic and discontinuous. It jumps from the constant value $10^{3}$ in the ring $\frac{1}{2 \sqrt{2}} \leq \mid x-$ $\mathbf{c} \mid \leq \frac{1}{2}, \mathbf{c}=\left(\frac{1}{2}, \frac{1}{2}\right)^{T}$, to 1 outside. We tested uniform grids with $n \times n$ nodes, $n=100,200,300,400$. The choice of the parameter $c$ is the same with Case $I$. Table 5 displays the results obtained by using three different preconditioners. The results are quite similar to the advection-diffusion problem. 
From Table 4 - 5] we can see that MTFFD is much more efficient; it only needs less than half of the iteration numbers that TFFD needs.

Case III: Skyscraper problems:

The tensor $\kappa$ is isotropic and discontinuous. The domain contains many zones of high permeability which are isolated from each other. Let $[x]$ denote the integer value of $x$. In $2 \mathrm{D}$, we have

$$
\kappa(x)=\left\{\begin{array}{l}
10^{3} *\left(\left[10 * x_{2}\right]+1\right), \quad \text { if }\left[10 * x_{i}\right]=0 \bmod (2), i=1,2 \\
1, \\
\text { otherwise }
\end{array}\right.
$$

The diagonal elements of $A$ jump between 4 and 36000. The parameter $c$ is chosen as 10 in the test. The numerical results are shown in table 6

Case IV: Convective skyscraper problems:

The same with the Skyscraper problems except that the velocity field is changed to be $\mathbf{a}=(1000,1000,1000)^{T}$. The diagonal elements of $A$ jump between 24 and 36020 . The parameter is chosen as 1 . The tested results are displayed in Table 7

From Table 6] - 7] we can see that Skyscraper and Convective skyscraper problems are quite difficult. The TFFD and ILU(0) preconditioned GMRES fail to converge for both problems. The MTFFD preconditioned GMRES has much better performance. For Skyscraper problem with $h=\frac{1}{400}$, we note that MTFFD needs 222 iterations to converge.

Case V: Anisotropic layers:

The domain is made of 10 anisotropic layers with jumps of up to four orders of magnitude and an anisotropy ratio of up to $10^{3}$ in each layer. The diagonal elements jump between 22 and 220000. The parameter $c$ is chosen as $\frac{2}{5}$. The test results are displayed in Table 8 From the table we can see that MTFFD preconditioner is much more efficient; as $h$ decreases, it needs only $\frac{1}{3}$ of the number of iterations that TFFD preconditioner needs.

Table 6: Test results for skyscraper problems, nonsymmetric matrix

\begin{tabular}{|r|r|r|r|r|r|r|}
\hline & \multicolumn{2}{|c|}{ MTFFD } & \multicolumn{2}{c|}{ TFFD } & \multicolumn{2}{|c|}{ ILU(0) } \\
\hline $1 / h$ & iter & error & iter & error & iter & error \\
\hline 100 & 151 & $8.8 \mathrm{e}-7$ & $\dagger$ & $1.3 \mathrm{e}-1$ & $\dagger$ & $1.1 \mathrm{e}-3$ \\
\hline 200 & 185 & $2.9 \mathrm{e}-6$ & $\dagger$ & $2.6 \mathrm{e}-1$ & $\dagger$ & $6.6 \mathrm{e}-3$ \\
\hline 300 & 159 & $6.2 \mathrm{e}-6$ & $\dagger$ & $3.9 \mathrm{e}-1$ & $\dagger$ & $8.6 \mathrm{e}-3$ \\
\hline 400 & $\dagger$ & $3.6 \mathrm{e}-4$ & $\dagger$ & $4.8 \mathrm{e}-1$ & $\dagger$ & $3.6 \mathrm{e}-2$ \\
\hline
\end{tabular}

In Figure 7 the eigenvalue distributions of the preconditioned matrices by TFFD and MTFFD preconditioners are displayed. The test matrices are generated from discretization of (31) with the above five different conditions and mesh size $h=\frac{1}{50}$. From the figures we can see that the MTFFD preconditioner can improve the eigenvalue distributions considerably. Particularly, the smallest eigenvalues are shifted in the positive direction, which makes the smallest eigenvalues to be well separated from the origin. The largest eigenvalues are remain very close to 1 . 
Table 7: Test results for convective skyscraper problems, nonsymmetric matrix

\begin{tabular}{|r|r|r|r|r|r|r|}
\hline & \multicolumn{2}{|c|}{ MTFFD } & \multicolumn{2}{c|}{ TFFD } & \multicolumn{2}{|c|}{ ILU(0) } \\
\hline $1 / h$ & iter & error & iter & error & iter & error \\
\hline 100 & 66 & $1.1 \mathrm{e}-8$ & $\dagger$ & $1.0 \mathrm{e}-4$ & 173 & $3.7 \mathrm{e}-8$ \\
\hline 200 & 94 & $9.2 \mathrm{e}-8$ & $\dagger$ & $4.3 \mathrm{e}-2$ & $\dagger$ & $1.1 \mathrm{e}-3$ \\
\hline 300 & 82 & $5.0 \mathrm{e}-8$ & $\dagger$ & $8.4 \mathrm{e}-2$ & $\dagger$ & $8.6 \mathrm{e}-3$ \\
\hline 400 & 133 & $4.1 \mathrm{e}-8$ & $\dagger$ & $2.9 \mathrm{e}-1$ & $\dagger$ & $4.5 \mathrm{e}-2$ \\
\hline
\end{tabular}

Table 8: Test results for anisotropic layers problems, nonsymmetric matrix

\begin{tabular}{|r|r|r|r|r|r|r|}
\hline & \multicolumn{2}{|c|}{ MTFFD } & \multicolumn{2}{c|}{ TFFD } & \multicolumn{2}{c|}{ ILU(0) } \\
\hline $1 / h$ & iter & error & iter & error & iter & error \\
\hline 100 & 29 & $3.1 \mathrm{e}-11$ & 68 & $1.4 \mathrm{e}-8$ & 190 & $3.6 \mathrm{e}-7$ \\
\hline 200 & 36 & $6.8 \mathrm{e}-7$ & 97 & $1.8 \mathrm{e}-8$ & $\dagger$ & $2.1 \mathrm{e}-4$ \\
\hline 300 & 40 & $2.3 \mathrm{e}-6$ & 120 & $1.2 \mathrm{e}-8$ & $\dagger$ & $2.8 \mathrm{e}-4$ \\
\hline 400 & 42 & $5.0 \mathrm{e}-6$ & 139 & $2.6 \mathrm{e}-8$ & $\dagger$ & $6.5 \mathrm{e}-3$ \\
\hline
\end{tabular}
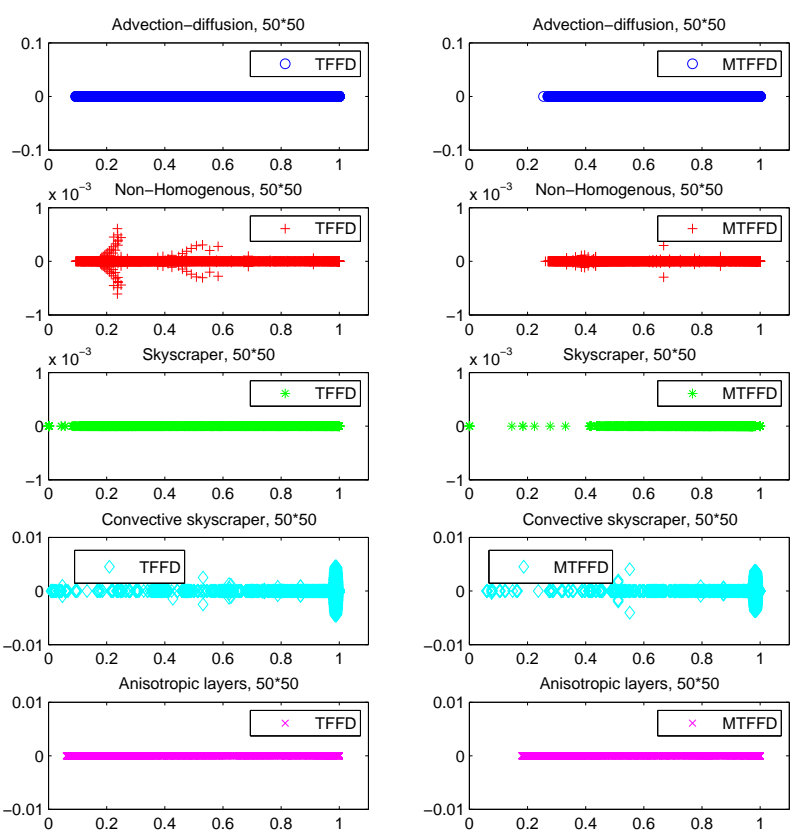

Figure 7: Spectrum distribution the preconditioned matrices. 
Example 2. We consider the constant-coefficient convection diffusion equation

$$
\begin{array}{lr}
-\Delta u+2 p_{1} u_{x}+2 p_{2} u_{y}-p_{3} u=f & \text { in }[0,1]^{2}, \\
u=g & \text { on } \partial[0,1]^{2},
\end{array}
$$

where $p_{1}, p_{2}$ and $p_{3}$ are positive constants. Discretization of the equation by the standard second order 5 -point stencil on a uniform $n \times n$ mesh gives rise to a sparse linear system

$$
A x=b
$$

where

$$
A=\operatorname{Btrid}_{n}(-(\beta+1) I, T,(\beta+1) I)
$$

and

$$
T=\operatorname{trid}_{n}(-\gamma-1,4-\sigma, \gamma-1),
$$

with $\beta=p_{1} h_{d}, \gamma=p_{2} h_{d}$ and $h_{d}=\frac{1}{n+1}$. The matrices series cdde1-cdde6 are based on the above equation with different parameters. We have tested all of the matrices, and the results are shown in Table 9 In the tests, the parameter $c_{d}$ is set to be 8 for cdde 3 and cdde 5 , and 1 for other matrices.

Table 9: Test results for the cdde series matrices, nonsymmetric matrices

\begin{tabular}{|r|r|r|r|r|r|r|}
\hline & \multicolumn{2}{|c|}{ MTFFD } & \multicolumn{2}{c|}{ TFFD } & \multicolumn{2}{|c|}{ ILU(0) } \\
\hline matrix $\left(p_{1}, p_{2}, p_{3}\right)$ & iter & error & iter & error & iter & error \\
\hline cdde1 $(1,2,30)$ & 32 & $6.4 \mathrm{e}-11$ & 197 & $3.4 \mathrm{e}-11$ & 50 & $5.0 \mathrm{e}-11$ \\
\hline cdde2 $(25,50,30)$ & 10 & $2.6 \mathrm{e}-11$ & 10 & $7.3 \mathrm{e}-12$ & 15 & $2.3 \mathrm{e}-12$ \\
\hline cdde3 $(1,2,80)$ & 42 & $5.9 \mathrm{e}-10$ & $\dagger$ & $3.4 \mathrm{e}-2$ & 62 & $3.0 \mathrm{e}-10$ \\
\hline cdde4 $(25,50,80)$ & 10 & $2.4 \mathrm{e}-11$ & 10 & $3.0 \mathrm{e}-12$ & 18 & $7.2 \mathrm{e}-12$ \\
\hline cdde5 $(1,2,250)$ & 68 & $1.5 \mathrm{e}-10$ & $\dagger$ & $1.8 \mathrm{e}-1$ & 96 & $5.6 \mathrm{e}-10$ \\
\hline cdde6 $(25,50,250)$ & 12 & $4.2 \mathrm{e}-12$ & 11 & $1.2 \mathrm{e}-11$ & 19 & $5.0 \mathrm{e}-12$ \\
\hline
\end{tabular}

From Table 9 we can see that the MTFFD preconditioner produces nearly the same results as that of the TFFD preconditioner for cdde2, cdde4 and cdde6. For the relatively difficult problems cdde1, cdde3 and cdde5, we can see that the MTFFD preconditioner is more efficient.

\section{Conclusions}

A modified tangential frequency filtering preconditioner is proposed and analyzed in this paper. The optimal order of modification and the optimal parameter are determined by the Fourier analysis. With the optimal order of modification, the results show that the preconditioned matrix has the condition number $\mathcal{O}\left(h^{-\frac{2}{3}}\right)$, which is much better than the BILU and MBILU precodnitioner. All the theoretical results are illustrated by the numerical tests. Finally, the performance of the new preconditioner is examined by some problems arising from discretization of PDEs with discontinuous coefficient. With the optimal order of modification, the major inconvenience of the present preconditioner is the choice of the relaxation parameter $c$, whose value is problem dependent. For future work, it may be worthwhile to investigate the idea of dynamically relaxed methods 25, 26, 32. This would hopefully further improve the robustness of the current preconditioner. 


\section{References}

[1] Y. Achdou and F. Nataf, An iterated tangential filtering decomposition, Numer. Linear Algebra Appl., 10, (2003), pp.511-539.

[2] Y. Achdou, F. Nataf, Low frequency tangential filtering decomposition, Numer. Linear Algebra Appl., 14, (2007), pp.129-147

[3] J. R. Appleyard and I. M. Cheshire, Nested Factorization, SPE 12264, presented at the Seventh SPE Symposium on Reservoir Simulation, San Francisco, 1983.

[4] O. Axelsson, A generalized SSOR method, BIT., 13, (1972), pp. 443-467.

[5] O. Axelsson and L. Kolotilina, Diagonally compensated reduction and related preconditioning methods, Numer. Linear Algebra Appl.,1, (1994), 155-177.

[6] O. Axelsson and V. A. Barker, Finite Element Solution of Boundary Value Problems, Theory and Computation., New York: Academic Press, 1984.

[7] O. Axelsson and H. Lu, On the eigenvalue estimates for block incomplete factorization methods, SIAM. J. Matrix Anal. Appl., 16, (1995), pp. 10741085 .

[8] O. Axelsson and G. Lindskog, On the eigenvalue distribution of a class of preconditioning methods, Numer. Math., 48, (1986), pp. 479-498.

[9] O. Axelsson, Iterative solution methods, Cambridge University Press, New York, 1994.

[10] T. Boonen, J. Van Lent and S. Vandewalle, Local Fourier analysis of multigrid for the curl curl equation, SIAM J. Sci. Comput., 30, (2008), pp.17301755 .

[11] A. Brandt, Rigorous local model analysis of Multigrid, Math. Comp., 31, (1977), pp. 333-390.

[12] A. Buzdin, Tangential decomposition, Computing., 61, (1998), pp.257-276.

[13] A. Buzdin and G. Wittum, Two-frequency decomposition, Numer. Math., 97, (2004), pp.269-295.

[14] A. Buzdin, D. Logashenko, and G. Wittum, IBLU decompositions based on Pade approximants, Numer. Linear Algebra Appl.,15, (2008), pp.717-746.

[15] T. F. Chan and H. C. Elman, Fourier analysis of iterative methods for elliptic problems, SIAM Review., 31, (1989), pp.20-49.

[16] T. F. Chan, Fourier analysis of relaxed incomplete factorization preconditioners, SIAM J. Sci. Comput., 12, (1991), pp.668-690.

[17] T. F. Chan and J. M. Donato, Fourier analysis of incomplete factorization preconditioners for three-dimensional anisotropic problems, SIAM J. Sci. Stat. Comput., 13, (1992), pp.319-338. 
[18] I. Chihiro, Fast solver for large systems of linear equations for finite element analysis on unstructured meshes, Ph.D thesis, Swinburne University of Technology, 2004.

[19] P. Concus, G. H. Golub and G. Meurant, Block Preconditioning for the Conjugate Gradient method, SIAM J. Sci. Statist. Comput., 6, (1985), pp.220252 .

[20] I. Gustafsson, A class of first order factorization methods, BIT., 18, (1978), pp.142-156.

[21] W. Hackbusch, Iterative solution of large sparse systems of equations, Springer, New York, 1994.

[22] B. N. Khoromskij, G. E. Mazurkevich and G. Wittum, Frequency filtering for elliptic interface probems with lagrange multipliers, SIAM J. Sci Comput., 21, (1999), pp. 421-440.

[23] R J. Le Veque and L. N. Trefethen, Fourier anlaysis of the SOR iterations, IMA J. Numer. Anal., 8, (1988), pp.273-279.

[24] H. Lu, Matrix compensation and diagonal compensation, J. Comput. Math. Appl., 63, (1995), 237-244.

[25] M. Magolu Monga-Made, Taking Advantage of the potentialities of dynamically modified block incomplete factorizations, SIAM J. Sci Comput., 19, (1998), pp. 1083-1108.

[26] M. Magolu Monga-Made, Dynamically relaxed block incomplete factorizations for solving two- and three-dimensional problems, SIAM J. Sci Comput., 21, (2000), pp. 2008-2028.

[27] The MathWorks, Inc. MATLAB 7, September 2004.

[28] J. A. Meijerink and H. A. van der Vorst, An iterative solution method for linear systems of which the coefficient matrix is symmetric M-matrix, Math. Comput., 137, (1977), pp.148-162.

[29] C. Mense and R. Nabben, On algebraic multilevel methods for nonsymmetric systems - comparison results Linear Algebra Appl. (2008), doi:10.1016/j.laa.2008.04.045.

[30] C. Mense and R. Nabben, On algebraic multilevel methods for nonsymmetric systems - convergence results, to appear in ETNA.

[31] R.B. Morgan and M. Zeng, Harmonic Projection Methods for Large Nonsymmetric Eigenvalue Problems, Numer. Linear Algebra Appl., 5, (1998) pp. 33-55.

[32] Y. Notay, DRIC: A dynamic version of the RIC method, Numer. Linear Algebra Appl.,1, (1994), 511-532.

[33] Y. Notay, Algebraic multigrid and algebraic multilevel methods: a theoretical comparison, Numer. Linear Algebra Appl. 2005; 12:419-451. 
[34] K. Otto, Analysis of preconditioners for hyperbolic partial differential equations, SIAM J. Numer. Anal. 33, (1996), pp.2131-2165.

[35] J.W. Ruge and K. Stüben, Algebraic Multigrid (AMG), In Multigrid Methods, Frontiers in Applied Mathematics, Vol 3, SIAM: Philadephia, PA, 1987, 73-130.

[36] Y. Saad, Iterative Methods for Sparse Linear Systems, PWS Publishing Company: Boston, MA, 1996.

[37] P. S. Vassilevski, Multilevel Block Factorization Preconditioners: Matrixbased Analysis and Algorithms for Solving Finite Element Equations, Springer, 2008.

[38] C. Wagner, Tangential frequency filtering decompositions for symmetric matrices, Numer. Math., 78, (1997), pp.119-142.

[39] C. Wagner, Tangential frequency filtering decompositions for unsymmetric matrices Numer. Math., 78, (1997), pp.143-163.

[40] C. Wagner and G. Wittum, Adaptive filtering, Numer. Math., 78, (1997), pp.305-382.

[41] R. Wienands, C. W. Oosterlee and T. Washio, Fourier analysis of GMRES(M) preconditioned by multigrid, SIAM J. Sci. Comput., 22, (2000), pp.582-603.

[42] G. Wittum, Filternde Zerlegungen, Schnelle Löser für große Gleichungssysteme. Teubner Skripten zur Numerik, Band 1, Teubner-Verlag, Stuttgart, 1992.

[43] G. Wittum, Shifted iterations, Numer. Math. 76, (1997), pp.265-278. 
Centre de recherche INRIA Saclay - Île-de-France

Parc Orsay Université - ZAC des Vignes

4, rue Jacques Monod - 91893 Orsay Cedex (France)

Centre de recherche INRIA Bordeaux - Sud Ouest : Domaine Universitaire - 351, cours de la Libération - 33405 Talence Cedex Centre de recherche INRIA Grenoble - Rhône-Alpes : 655, avenue de l'Europe - 38334 Montbonnot Saint-Ismier

Centre de recherche INRIA Lille - Nord Europe : Parc Scientifique de la Haute Borne - 40, avenue Halley - 59650 Villeneuve d'Ascq Centre de recherche INRIA Nancy - Grand Est : LORIA, Technopôle de Nancy-Brabois - Campus scientifique 615, rue du Jardin Botanique - BP 101 - 54602 Villers-lès-Nancy Cedex

Centre de recherche INRIA Paris - Rocquencourt : Domaine de Voluceau - Rocquencourt - BP 105 - 78153 Le Chesnay Cedex

Centre de recherche INRIA Rennes - Bretagne Atlantique : IRISA, Campus universitaire de Beaulieu - 35042 Rennes Cedex

Centre de recherche INRIA Sophia Antipolis - Méditerranée : 2004, route des Lucioles - BP 93 - 06902 Sophia Antipolis Cedex 